\title{
Structural variation and the plant pan genomes
}

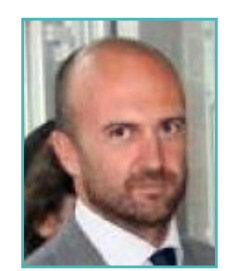

The analysis of variation in plants has revealed that their genomes are characterised by high levels of structural variation, consisting of both smaller insertion/deletions, mostly due to recent insertions of transposable elements, and of larger insertion/ deletion similar to those termed in humans Copy Number Variants (CNVs). These observations indicate that a single genome sequence might not reflect the entire genomic complement of a species, and prompted us to introduce the concept of the plant pan-genome, including core genomic features common to all individuals and a Dispensable Genome (DG) composed of partially shared and/or non shared DNA sequence elements. The very active transposable element systems present in many plant genomes may account for a large fraction of the DG. Both the mechanisms by which the CNV-like variants are generated and the direction of the mutational events are still unknown. Uncovering the intriguing nature of the DG, i.e. its composition, origin and function, represents a step forward towards an understanding of the processes generating genetic diversity and phenotypic variation. Additionally, since the DG clearly appears to be for the most part the youngest and most dynamic component of the pan genome, it is of great interest to understand whether it is a major contributor to the creation of new genetic variation in plant evolution as well as in the artificial selection processes of plant breeding. We will discuss the extent and composition of the pan genome in different plant species, the different mechanisms that generate and maintain the dispensable portion, the phenotypic effects of the DG and the rates and modes of creation of new genetic variation due to DG components. 